\title{
Investigating Swirl and Tumble Flow with a Comparison of Visualization Techniques
}

\begin{abstract}
Robert S. Laramee* VRVis Research Center, Vienna
\end{abstract}

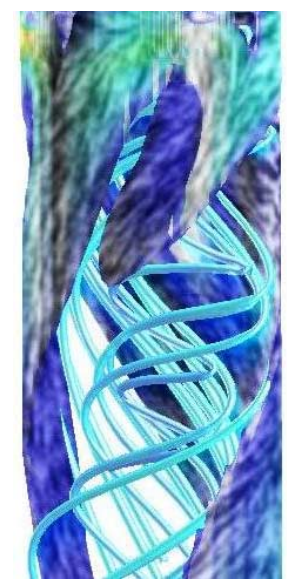

\author{
Daniel Weiskopf $\$$ \\ VIS, University of Stuttgart
}

\author{
Jürgen Schneider ${ }^{\mathrm{Il}}$ \\ AVL, Graz
}

Helwig Hauser*
VRVis Research Center, Vienna
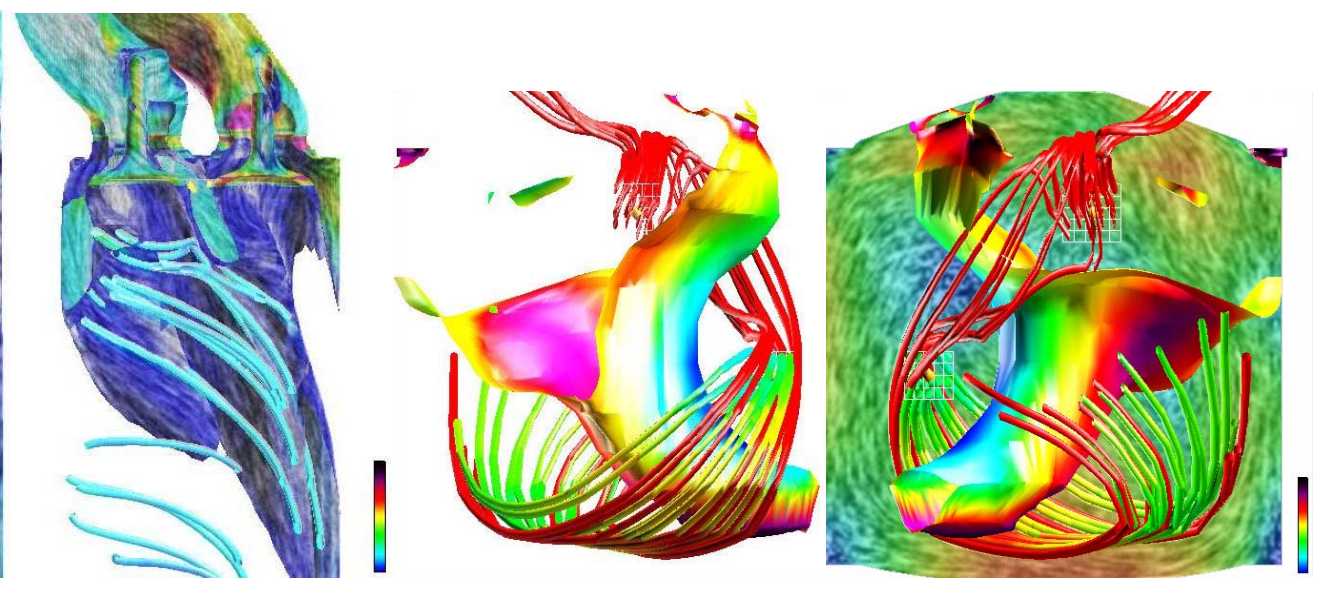

Figure 1: Visualization of swirl and tumble flow using a combination of direct color-mapping, streamlines, isosurfaces, texture-based flow visualization and slicing. (Left) visualizing swirl flow using 3D streamlines and texture-based flow visualization on an isosurface, (middle-left) a clipping plane is applied to reveal occluded flow structures, (middle-right) an isosurface and 3D streamlines visualize tumble motion, and (right) the addition of texture-based flow visualization on a color-mapped slice.

\begin{abstract}
We investigate two important, common fluid flow patterns from computational fluid dynamics (CFD) simulations, namely, swirl and tumble motion typical of automotive engines. We study and visualize swirl and tumble flow using three different flow visualization techniques: direct, geometric, and texture-based. When illustrating these methods side-by-side, we describe the relative strengths and weaknesses of each approach within a specific spatial dimension and across multiple spatial dimensions typical of an engineer's analysis. Our study is focused on steady-state flow. Based on this investigation we offer perspectives on where and when these techniques are best applied in order to visualize the behavior of swirl and tumble motion.
\end{abstract}

CR Categories: I.3.3 [Computer Graphics]: Picture/Image Generation; I.3.7 [Computer Graphics]: Three-Dimensional Graphics and Realism-Color, shading, shadowing, and texture I.6.6 [Simulation and Modeling]: Simulation Output Analysis

Keywords: Flow visualization, computational fluid dynamics (CFD), swirl flow, tumble flow, visualization systems, engine simulation, in-cylinder flow

\section{INTRODUCTION}

The VRVis Research Center collaborates with AVL (www.avl.com) in order to provide visualization solutions for analysis of their CFD

*email: \{Laramee,Hauser $\} @$ VRVis.at

§email: weiskopf@vis.uni-stuttgart.de

Ilemail: juergen.schneider@avl.com simulation results. AVL's own engineers as well as engineers at industry affiliates use visualization software to analyze and evaluate the results of their automotive design and simulation.

Previously, AVL engineers used a series of several color-mapped slices to assess and visualize the results of their CFD simulations. Isosurfaces were used less commonly to assess certain 3D features that could not be investigated sufficiently with 2D slices. Recently, new solutions for the visualization of CFD simulation data have been introduced. We report on the application of these techniques in addition to the more traditional approaches. In this paper we describe: (a) the application of different visualization techniques to specific application cases, (b) advantages and disadvantages of what these techniques offer, and (c) a comparison which may apply to other application cases. We also to give recommendations on when to use specific techniques and in which application scenario.

\section{Evaluating Swirl and Tumble Motion}

For many of the automotive components that undergo evaluation, there is an ideal pattern of flow the engineers are trying to create. In the flow within a cylinder, we can distinguish between two types of motion: swirl flow commonly found in diesel engines and tumble flow commonly found in gas engines. In both cases, rotational motion occurs about an axis, though the position of the respective axis is different. In the case of swirl flow, the axis is more or less coincident with the cylinder axis, as shown in Figure 2. In the case of tumble (Figure 3), the rotation axis is perpendicular to the cylinder axis and more complex, thus making tumble flow more difficult to control than swirl flow. We refer to the rotational axis associated with tumble motion as the $z$ axis that, in this case, points out of the paper towards the reader.

In order to generate swirl or tumble motion, fluid enters the combustion chamber from the intake ports. Later on in the engine cy- 

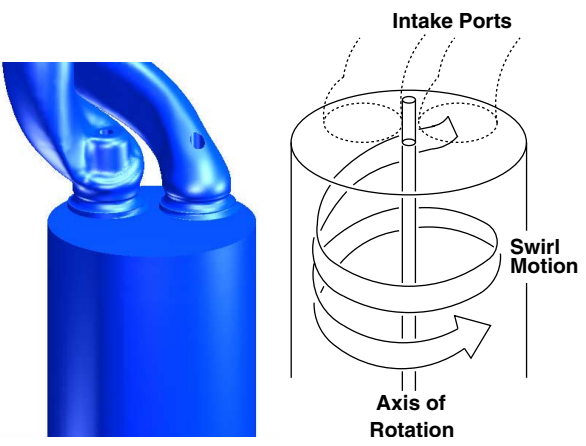

Figure 2: The swirling motion of flow in the combustion chamber of a diesel engine. Swirl is used to describe circulation about the cylinder axis. The intake ports at the top provide the tangential component of the flow necessary for swirl. The data set consists of 776,000 unstructured, adaptive resolution grid cells.

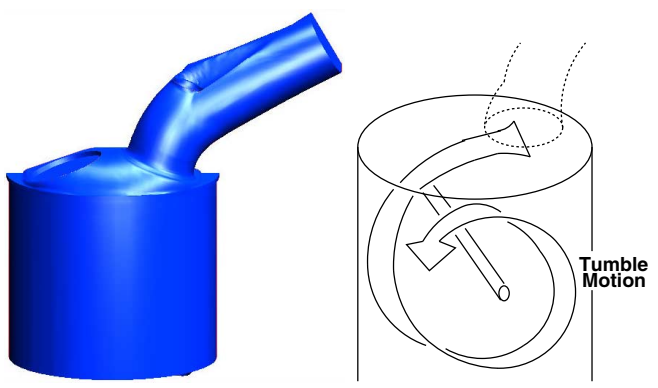

Figure 3: Some gas engine components require a tumble motion flow pattern (right) in order to mix fluid with oxygen. Tumble flow circulates around an axis perpendicular to the cylinder axis, orthogonal to swirl flow. The data set is composed of 61,700 unstructured, adaptive resolution cells.

cle, the kinetic energy associated with this motion is used to generate turbulence for mixing of fresh oxygen with evaporated fuel. The more turbulence generated, the better the mixture of air and fuel, and thus the more stable the combustion itself. By stable we mean achieving the same conditions for each engine cycle. Ideally, enough turbulent mixing is generated such that $100 \%$ of the fuel is burned. The swirl or tumble motion should be maximized to maximize turbulence. From the point of view of the mechanical engineers designing the intake ports, the ideal flow pattern leads to beneficial conditions including: improved mixture preparation, a higher EGR (Exhaust Gas Ratio) which means a decrease in fuel consumption, and lower emissions. However, too much swirl (or tumble) can displace the flame used to ignite the fuel, cause irregular flame propagation, or result in less fuel combustion.

As such, a balance must be achieved between generating enough swirl or tumble flow and not displacing the flame used to ignite the flow. A controlled flow motion is used to get stable and reproducible conditions at each engine cycle.

\subsection{Investigating Flow Patterns with Visualization}

Central to this study are some routine questions that engineers may ask when investigating swirl and tumble flow:

1. Can visualization provide insight into or verify the characteristic shape and behavior of the flow?

2. What tools can help to visualize the swirl and tumble flow patterns?
3. Where in the combustion chamber are the ideal swirl and tumble flow pattern not being realized?

Here we seek answers to some of these fundamental questions using direct, geometric, and texture-based flow visualization techniques. We proceed with a visual analysis of the simulation data from AVL's CFD solver. In particular, we analyze the simulation data from two important in-cylinder flow motion patterns, typical of a mechanical engineer's analysis. Our investigation also carries forth across multiple spatial dimensions, namely in $2 \mathrm{D}, 2.5 \mathrm{D}$, and $3 \mathrm{D}$. By $2.5 \mathrm{D}$ we mean surfaces through $3 \mathrm{D}$ space. We note that the large size of the data set shown in Figure 2, 776,000 unstructured, adaptive resolution cells, makes unsteady flow visualization very difficult with our hardware.

\subsection{Direct, Geometric, and Texture-Based Flow Visualization}

Three different approaches are widely used in flow visualization:

Direct flow visualization: This category of techniques uses a translation that is as direct as possible for representing flow data in the resulting visualization. The result is an overall picture of the flow e.g., Figure 4, top-left. Common approaches are drawing arrows or color coding velocity. Intuitive pictures can be provided, especially in the case of two dimensions.

Geometric flow visualization: These approaches often first integrate the flow data and use geometric objects in the resulting visualization. The objects have a geometry that reflects the properties of the flow. Examples include streamlines, streaklines, and timelines. Not all geometric objects are based on integration. Another useful geometric approach is generating isosurfaces, e.g., with respect to an isovalue of pressure or magnitude of velocity. A more thorough description of geometric techniques is presented by Post et al. [11]

Dense, texture-based flow visualization: A texture is computed that is used to generate a dense representation of the flow, e.g., Figure 4, bottom-right. A notion of where the flow moves is incorporated through co-related texture values along the vector field. In most cases this effect is achieved through filtering of texels according to the local flow vector. Texture-based methods offer a dense representation of the flow with complete coverage of the vector field. In this paper we use advection approaches according to Image Based Flow Visualization (IBFV) [19] and Image Space Advection (ISA) [9], which can generate both Spot Noise [18] and LIC-like [3] imagery. These approaches are related to LagrangianEulerian Advection (LEA) [6]. We note that a full comparison of texture-based flow visualization techniques [8] is beyond the scope of this paper.

We focus only on interactive visualization techniques because an interactive exploration of parameter space is essential for improving the design of intake ports and valve cylinders (e.g., changing isovalues or placing seed points of particles). Hence feature-based flow visualization, another class of techniques including feature extraction and tracking is not a focus of ours. Another problem is that each engine component has its own characteristic flow motion. Implementing a feature extraction algorithm for each flow pattern is simply not practical. Further complicating the matter is that the ideal flow motion is only approximated. Post et al. [12] cover feature-based flow visualization in detail.

The rest of the paper is organized as follows: Sections 3, 4, and 5 investigate properties of the flow patterns using 2D slices, surfaces (referred to as $2.5 \mathrm{D}$ ), and $3 \mathrm{D}$ and hybrid techniques respectively. Each spatial domain is analyzed using direct, geometric, and texture-based visualization tools. These approaches are then compared to one another. Section 6 presents a discussion and offers some overall perspectives with respect to this investigation and Section 7 outlines our conclusions. 

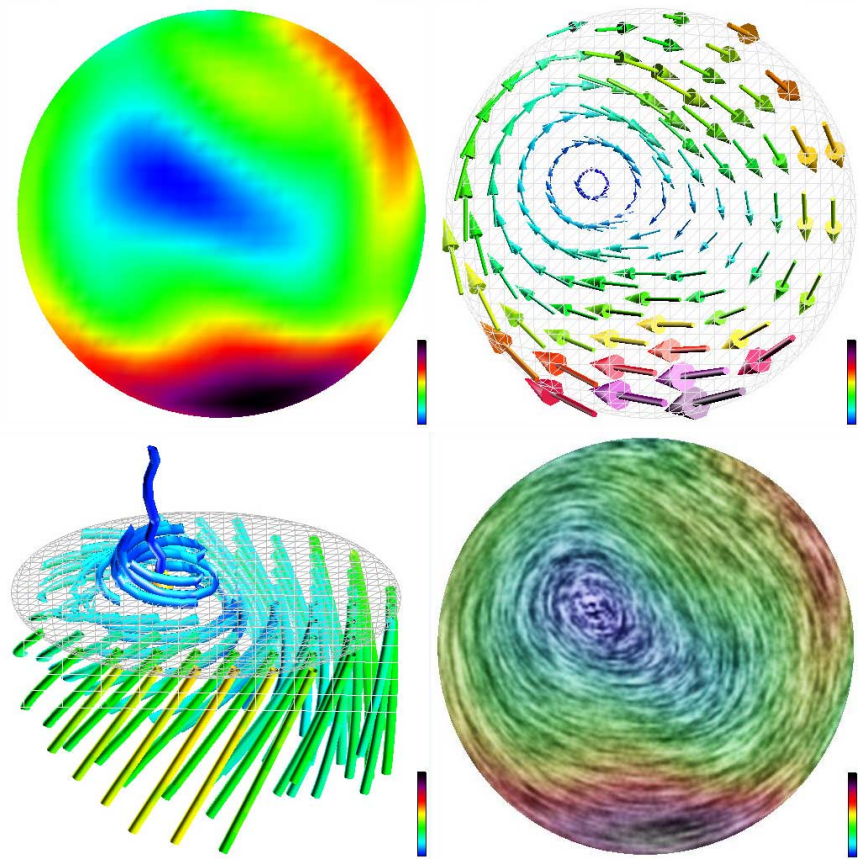

Figure 4: The visualization of swirl flow on a slice through the combustion chamber of a diesel engine: (top-left) direct visualization using color mapping, (top-right) direct visualization using glyphs, (bottom-left) geometric flow visualization using streamlets, and (bottom-right) texture-based flow visualization.

\section{Visualizing Flow Motion on 2D Slices}

Slices are a common tool used to investigate the properties of the flow inside a volume. One reoccurring theme is that of placement: We must decide where to slice through the volume. This decision is influenced by two factors: (1) our knowledge and experience regarding the data set and (2) some trial and error. The average user in this case is a mechanical engineer with a strong background and previous training in the area of CFD. These users generally have a priori knowledge about the data they are investigating. In other words, they already have an idea and associated expectations of the behavior they would like to see. Nevertheless, after an initial slice is generated, further refinement of the visualization parameters might be needed for the desired results.

\subsection{Direct Flow Visualization}

Figure 4 shows a 2D slice through the cylindrical combustion chamber from Figure 2, within a plane perpendicular to the axis of rotation. In this particular case, each approach indicates that the flow has desirable properties, namely, a rotational pattern about the axis parallel to the cylinder, consistent with swirl motion.

Figure 4 top-left shows velocity magnitude mapped to hue. The colors help to differentiate regions of the flow and give an overview of the data. However, the path of the flow is not conveyed clearly. Figure 4 top-right shows shows the same data visualized with 3D glyphs placed on a $2 \mathrm{D}$ slice. In this case, the data has been resampled onto a regular grid with a user-specified resolution. A brute-force hedgehog technique results in visual clutter and over complexity. We have used the resampling tool to create a polar grid and interactively position the center of the grid such that it aligns with the vortex associated with the swirl flow. The glyphs give an overview of the vector field, show the direction of the flow, and also afford the visualization of the normal component of the flow with respect to the slice, an ability important for applications from the
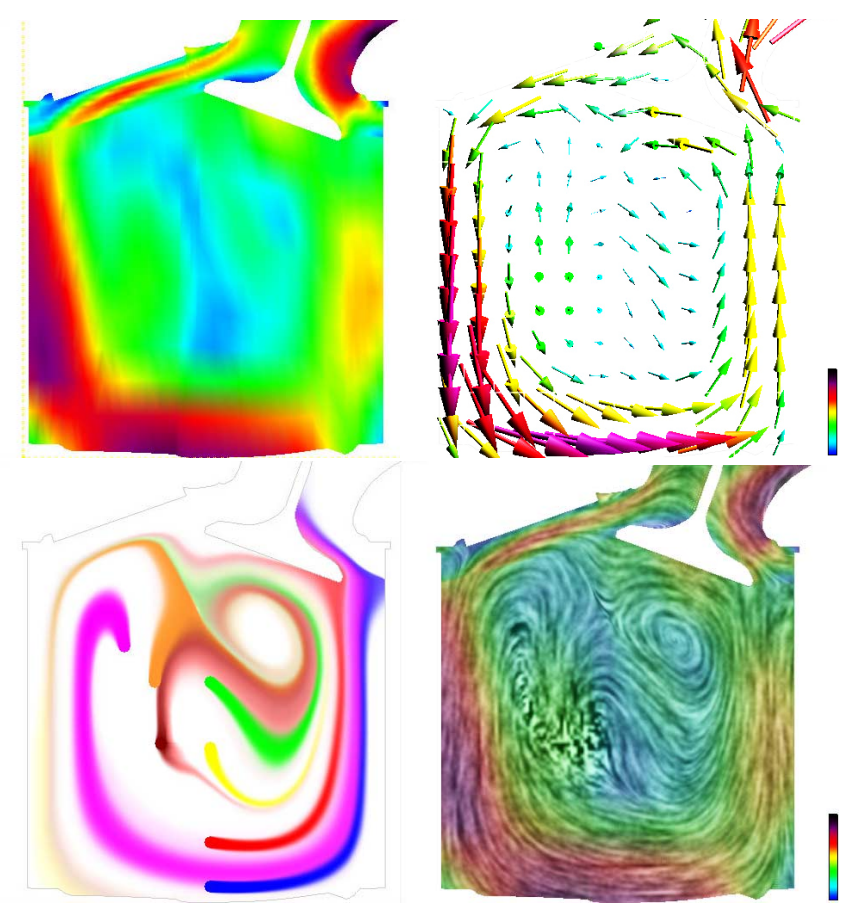

Figure 5: The visualization of tumble flow on a slice: (top-left) direct visualization using color mapping, (top-right) direct visualization using glyphs, (bottom-left) flow visualization using dye injection, and (bottom-right) texture-based flow visualization.

automotive industry [14]. This third component is missing from two of the other visualizations. However, what the glyphs lack is spatial continuity. There is always a trade-off between the resolution of resampling grid and coverage of the flow. Higher resolution resampling decreases the likelihood of overlooking a feature of the vector field, but increases perceptual problems.

Figure 5 top, shows direct visualization techniques applied to a slice in order to visualize tumble flow. The glyphs suggest that the flow pattern on the slice is generally consistent with tumble flow. We see an overall circular, counter-clockwise flow about an axis pointing out of the plane. However, some of the features of the flow are higher in spatial frequency that the glyph placement provided by the resampler. If we increase the resampling resolution, the result is difficult to interpret because the glyphs collide in visual space. Color coding, as in Figure 5 top-left, is much less suitable for tumble flow than for swirl flow because the structure of the flow is more complex and cannot be visualized by the magnitude of the velocity.

\subsection{Geometric Flow Visualization}

In general, geometric techniques like streamlines provide more spatial continuity than glyphs or texture-based flow visualization. Texture-based methods require a long convolution filter length to achieve similar spatial continuity. For the case of swirl flow, we find 2D streamlines restricted to the slice misleading because there is a strong flow component orthogonal to the slice, i.e., these long streamlines have less correspondence to physical motion. Hence, Figure 4 bottom-left shows the vector field visualized with 3D streamlets, namely, short streamlines. These streamlets show the strong component of the flow orthogonal to the slice and introduce more spatial continuity than the glyphs. However, while streamlets do increase spatial continuity they share the same disadvantage as the glyphs in terms of seeding and perceptual challenges.

Figure 5 bottom-left shows tumble flow on slice visualized using dye injection. The result resembles streaklines which are the 
same as streamlines for the case of steady-state flow. This particular implementation is done via dye injection in a manner similar to IBFV. The result has some characteristics from both the geometric and texture-based classes of techniques. The borderlines between dye color and white are geometric in the sense that they meet the definition of a streamline: a geometry that is everywhere tangent to the flow. On the other hand, the implementation is realized using textures which introduces diffusion into the result. Hence this technique could also be classified as texture-based.

The advantages here are that these objects show the downstream and upstream direction of the flow especially clear in an animation and the user may interactively select the seed points and dye color. Hence, the user may use these dye sources to highlight certain areas of the flow such as the vortex associated with the swirl flow. In this case, the flow component normal to the slice is not as strong compared with Figure 4 . The disadvantage here, and with geometric techniques in general is that placement is crucial. Important features maybe overlooked depending on the spatial position of the objects. Also we must use caution when interpreting these results because no particle actually traverses a path shown in Figure 5. The original CFD simulation data is $3 \mathrm{D}$ and time-dependent. We note that in this study, we focus on steady-state flow.

\subsection{Texture-Based Flow Visualization}

Figure 4 bottom-right shows the swirl motion with texture-based flow visualization applied. The advantages of this approach include: (1) complete coverage of the flow field, (2) spatial continuity, (3) velocity magnitude may be encoded in the texture, leaving hue available to include another CFD simulation attribute such as temperature, and (4) flow orientation (upstream and downstream direction) is clear in an animated sequence. In general, computation time is a disadvantage with many texture-based flow visualization techniques. Recently, this hurdle has been overcome with some techniques $[9,10,22,19]$. However, this approach does not generally provide as much spatial continuity as geometric techniques.

In Figure 5 both the dye injection and the texture-based flow visualization techniques reveal that the vector field has properties that deviate from the ideal tumble flow pattern. These techniques indicate the presence of a saddle point in the flow near the top, center of the geometry (See Helman and Hesselink [5] or Post et al. [12] for a more thorough description of flow topology). The deviance from the ideal tumble motion is a result of a trade-off made in the design of the intake port, as its shape must also allow enough incoming fresh air to mix with fuel. Thus air is let in from both sides of the intake port (left and right in this slice). The ideal tumble flow corresponds more closely to one central vortex in the flow. The orange and green dye sources can help us to highlight the saddle point while the texture-based approach ensures that the feature is not overlooked by providing a result with a dense representation across the complete slice.

We also note that the geometric and texture-based visualizations highlight the important asymmetric nature of the flow. There is a counter-clockwise looping pattern near the boundary of the geometry. This is especially clear in an animation. If the flow were too symmetric, the looping pattern might disappear leading to too much destructive flow motion.

\section{SWirl and Tumble Flow Visualized on Surfaces}

Examining the properties of the flow on boundary surfaces (referred to also as $2.5 \mathrm{D}$ ) is useful because we sometimes start our investigation at the surface in order to get an overview of the vector field before looking inside the volume.

\subsection{Direct Flow Visualization}

Figure 6 shows the flow at the surface from the diesel engine component from Figure 2. In this case, color mapping alone already

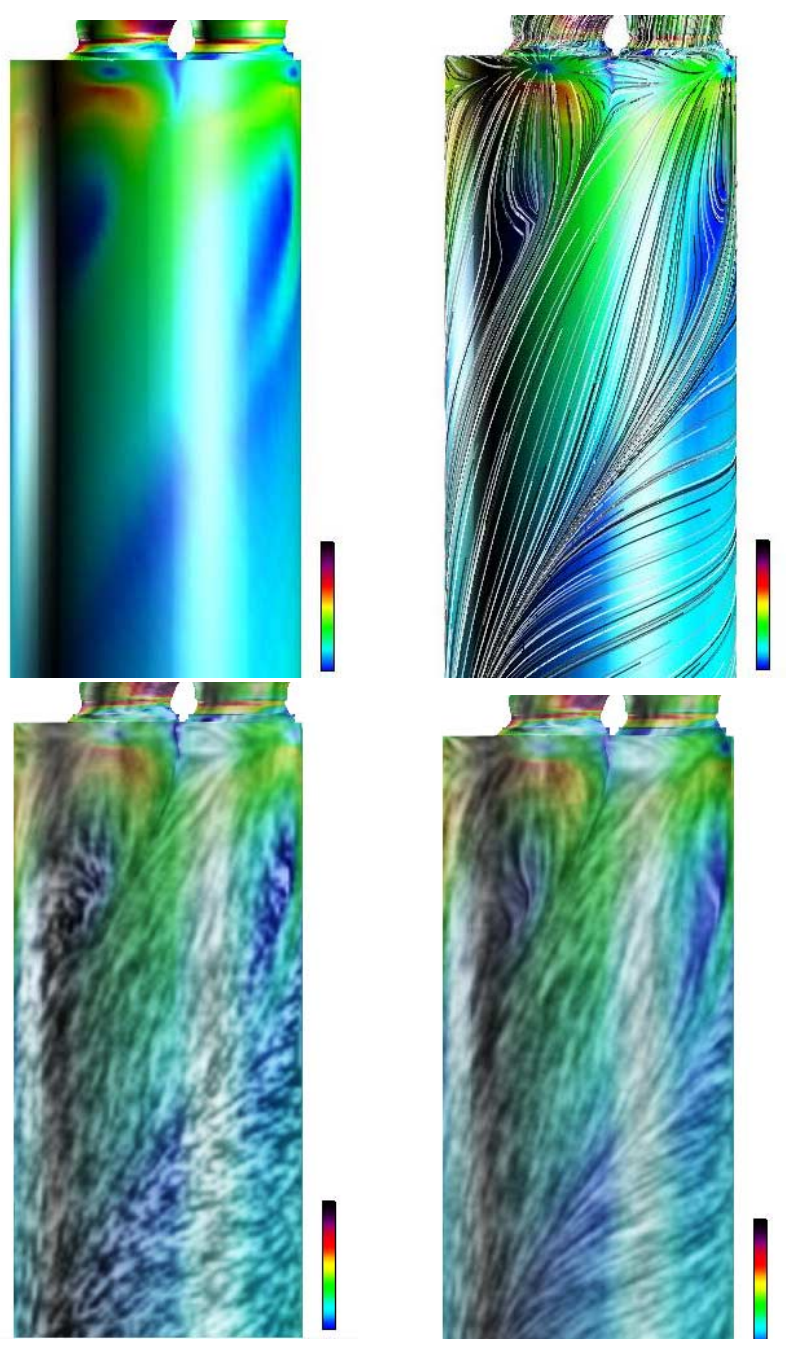

Figure 6: The visualization of swirl flow at the boundary surface of the combustion chamber: (top-left) direct visualization using color mapping, (top-right) geometric flow visualization using streamlines, (bottom-left) spot noise-like texture-based flow visualization, and (bottom-right) LIC-like texture-based flow visualization in combination with a velocity clamp.

reveals that the flow has swirl motion characteristics at this instant in time. We see regions of color forming a loose spiral pattern consistent with Figure 2.

Figure 7 shows the visualization of tumble flow at the surface. From color-mapping alone, it is difficult to answer our questions from Section 2.1. The pattern of flow is more complicated than in the case of swirl. However, color-mapping is still useful in order to identify extremal properties of the simulation data, such as very low velocity magnitude, characteristics that we are generally very interested in avoiding.

\subsection{Geometric Flow Visualization}

Figure 6 top-right illustrates the use of streamlines in order to visualize the properties at the surface. In this case, the implementation computes explicit integral paths according to a particle tracing algorithm. We have added a gray scale phase shift to the hue of each streamline to distinguish individual streamlines that collide. The streamlines are useful in illustrating the swirl behavior at the surface explicitly. Plus, they help the user to discover an important 

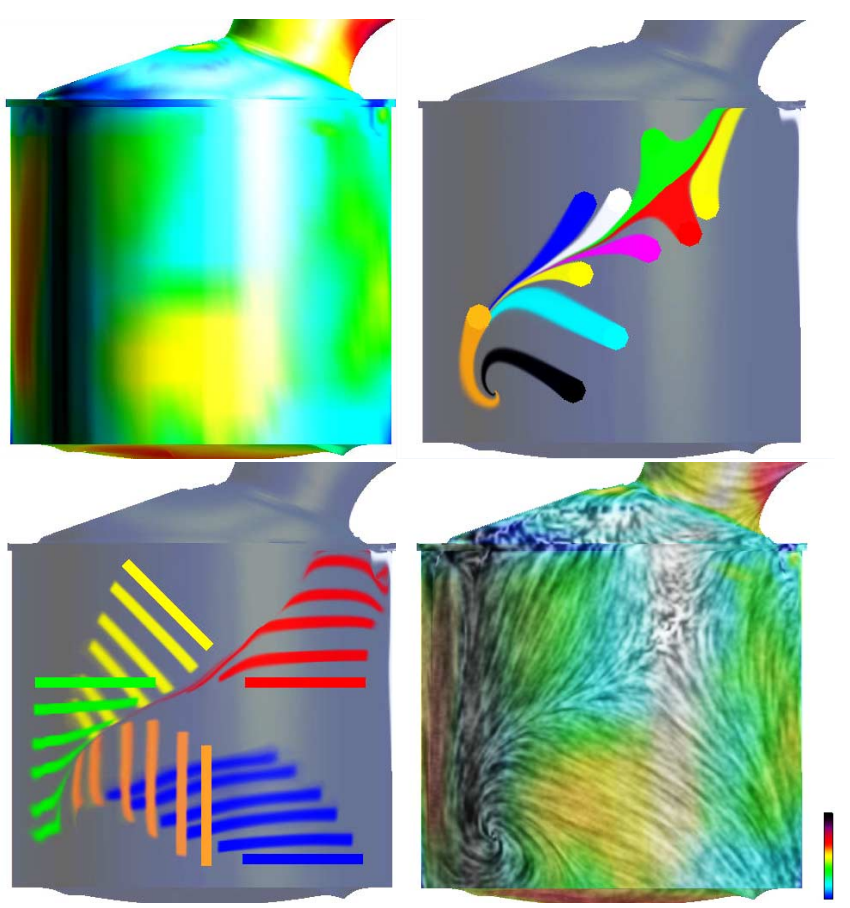

Figure 7: The visualization of tumble flow at the boundary surface: (top-left) direct visualization using color mapping, (top-right) visualization using dye injection, (bottom-left) geometric flow visualization using timelines, and (bottom-right) texture-based flow visualization.

area where the ideal swirl flow pattern is not being met, namely in the top-center of the geometry just below the intake ports. Here, destructive flow is evident: two regions of flow working against one another. However, caution must be used in the interpretation of this approach. Technically, the velocity has zero magnitude at the surface. What is shown is the velocity just under the surface, extrapolated and projected. No particle actually traverses an entire path shown by a streamline in this case.

Figure 7 illustrates streamlines and timelines, used to investigate the behavior of tumble flow at the surface. The streamlines (Figure 7 top-right), again implemented via dye injection as described in Section 3.2, have been seeded in order to selectively highlight a subset of the vector field topology, namely, a separatrix-a streamline between two critical points in the vector field [4]. This particular separatrix is especially visible between the green and red dye sources. Unfortunately, this is an undesirable feature of the flow for the case of tumble flow.

Timelines are the lines joined by a set of massless particles released into the flow at the same time. The dye-injected emulation of timelines shown in Figure 7 bottom-left are released from userspecified locations and orientations. In our implementation, the user may slide, scale, and rotate the release mechanism in addition to specifying color. Allowing the user to specify color is important in order to match correlated timelines. This particular set of timelines is helpful in visualizing convergent (indicated by the yellow and green timelines) and divergent (indicated by the blue timeline) areas of the surface flow. Ideally, the flow emerging from an intake port exhibits divergent behavior.

\subsection{Texture-Based Flow Visualization}

Texture-based visualization is very useful for generating an overview of the flow behavior at the surface. The advantage is that we obtain complete coverage of the flow and maintain spatial coherency according to the flow simultaneously. Plus, flow orienta- tion (upstream vs. downstream flow) may be visible in a still image when a spot noise-like texture is used, although the flow orientation is much clearer in an animated sequence. If we indicate velocity magnitude by the amount of smearing in the texture, as in Figure 6 bottom-left, than we can encode another CFD attribute as hue such as temperature. On the other hand, it is more difficult to see the properties of the flow in areas of low velocity magnitude. We can address this by applying a velocity clamp as shown in Figure 6 bottom-right. The result resembles LIC. Again, the destructive flow pattern is very noticeable with a texture-based approach. This is especially clear in a close-up view of the surface. We also note that the interpretation of the results shown in this case may be considered less misleading since long, explicit particle tracing paths are not depicted.

In Figure 7 bottom-right, we can see that the vortex that characterizes the ideal tumble motion is off-center in the lower-left of the geometry whereas the ideal tumble motion is characterized by a vortex in the center about the $z$ axis pointing towards the viewer. In the next section, we show how to visualize this vortex in $3 \mathrm{D}$.

\section{3D AND HYBRID APPROACHES}

Slices are commonly used to investigate the properties of the flow inside the volume but visualizing 3D characteristics of the flow like swirl can be difficult with 2D slices. We are interested in visualization techniques that provide insight into the spatial dimension orthogonal to the slice as well. In this section we discuss 3D and hybrid approaches. In this case, hybrid approaches are both a mixture of spatial dimensions, e.g., 2.5D and 3D as well was a mixture of flow visualization classifications such as geometric and texturebased flow visualization.

\subsection{Direct Flow Visualization}

Direct flow visualization techniques are generally difficult to apply in 3D. Figure 8 left shows 3D swirl flow visualized using colormapped vector glyphs. This image suffers from perceptual problems such as occlusion, visual complexity, and lack of depth cues. Furthermore, this is not a brute-force hedgehog approach. A subset of the 3D combustion chamber data has been resampled onto a regular polar grid similar to Figure 4. Even with the resampling approach, many of the individual glyphs are occluded by larger glyphs or too small to see clearly. The consequence is that we usually view only a subset of the $3 \mathrm{D}$ data.

\subsection{Geometric Flow Visualization}

Without special handling, geometric techniques can also suffer from some of the same perceptual problems that direct flow visualization can. One means by which to focus on a particular subset, area of interest, or feature of a flow field is via a streamline seeding strategy. In general, three popular streamline seeding strategies are often used: (1) image-based seeding strategies such as that described by Turk and Banks [17] or the evenly spaced-streamline seeding strategy presented by Jobard and Lefer [7], (2) topological or feature-based, seeding strategies such as those presented by Verma et al. [21] or Sanna et al. [13], or (3) interactive seeding strategies using a streamline seeding rake used by Bryson and Levit [2] or Schultz et al. [15]. Our approach falls into the third category-an interactive streamline seeding strategy.

A schematic of our interactive streamline seeding tool is shown in Figure 9. This tool provides the user with six interactive degrees of freedom (DoF): (1-3) three translational, (4) scaling, (5) rotational, and (6) resolution control. In addition, the containing plane can be chosen either as the $X Y, X Z$, or $Y Z$ plane. These interactive DoFs are required to investigate the results of CFD simulations because the meshes from CFD embrace a wide variety of components, features, and levels of resolution. Ideally, the tools used to analyze and visualize these data sets should be flexible enough to 

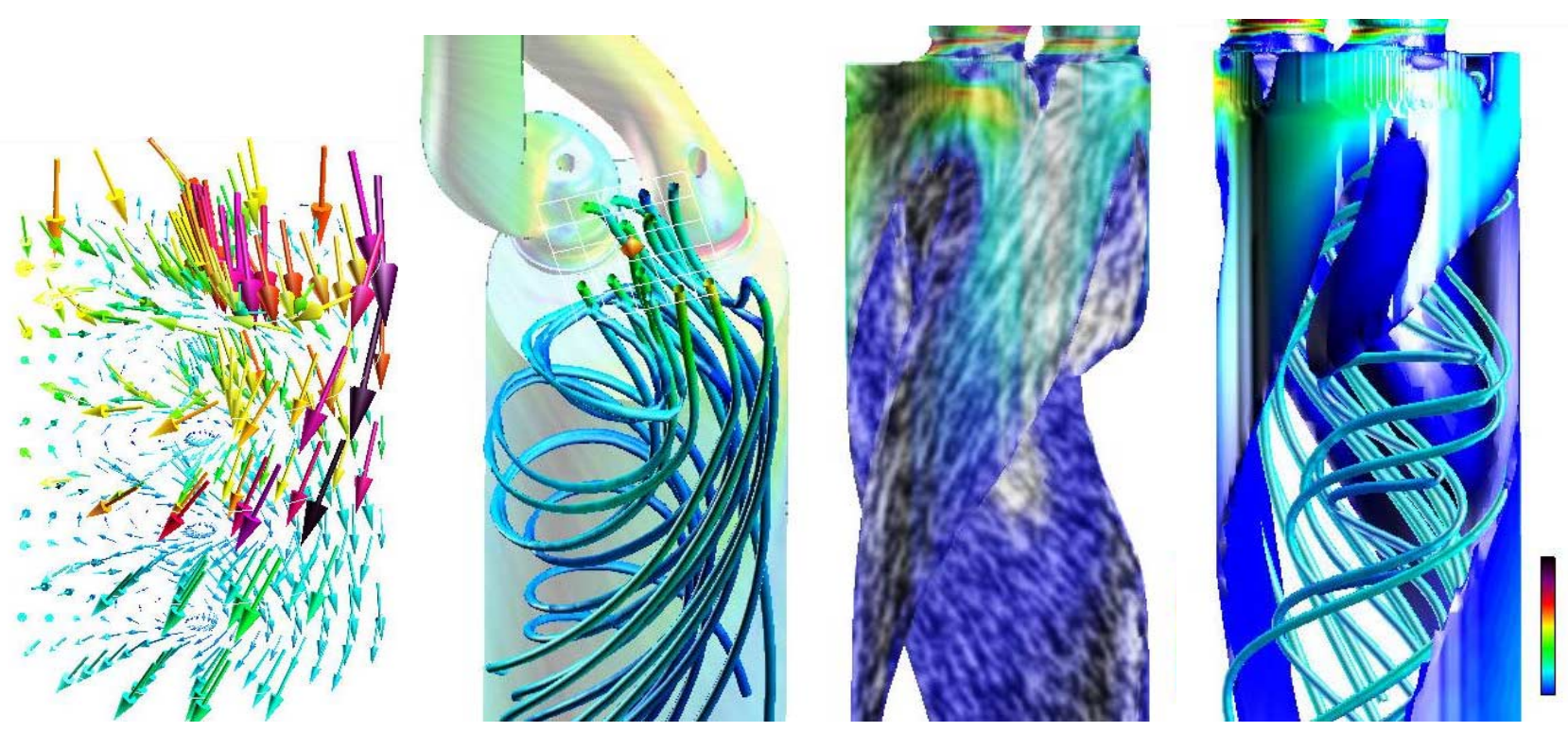

Figure 8: The visualization of swirl flow in the volume of the the combustion chamber: (left) direct visualization using color-mapped glyphs, (middle-left) geometric flow visualization using color-mapped shaded streamlines, (middle-right) texture-based flow visualization on a velocity isosurface, and (right) isosurfacing combined with 3D streamlines.

adapt their size, orientation, and resolution to fit the features of interest either automatically or through user-specified parameters. Figure 8 middle-left, shows streamlines color-mapped with veloc-

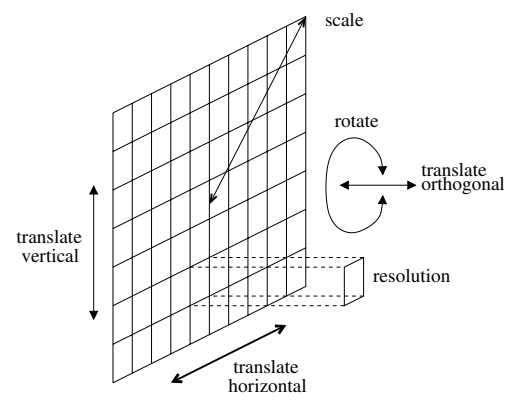

Figure 9: Our seeding plane implementation has six interactive DoFs: (1-3) three translational, (4) scaling, (5) rotation, (6) resolution.

ity magnitude, placed according to seeding plane in order to visualize the 3D swirl flow. We have employed an approach where a semi-transparent, velocity color-mapped surface serves as context information. In this case, the streamlines show the swirling behavior of the flow rather clearly. The main behavior of the flow appears to match the sought after swirl flow pattern.

Swirl flow is more stable than tumble flow, one reason being that the axis of rotation and the cylinder axis are aligned with swirl motion. In tumble motion, these two axes are orthogonal making it more difficult to realize and visualize. Figure 10 left shows the visualization of tumble flow with streamlines seeded by two seeding planes. A 2D slice with a gray-scale velocity magnitude color map is used for context. The top-center seeding plane uses streamlines color-mapped according to velocity magnitude. The bottom-left seeding plane uses red streamlines only in order to help distinguish seeding plane-specific streamlines. The bottom-left seeding plane also uses a tool that lets the user interactively control the length of the streamlines. In this case, the tool has been used to truncate the geometry in order to reduce occlusion and visual complexity.

\subsection{Texture-Based Flow Visualization}

In a hybrid visualization approach, we apply ISA for producing dense, texture-based representations of flow on isosurfaces. Isosurfaces are a visualization tool routinely used to investigate the properties of the flow inside a 3D volume. The shape of an isosurface can give us insight into its 3D characteristics.

Figure 8 right shows two geometric approaches combined, namely, color-mapped streamlines and a velocity isosurface. Applying texture-based flow visualization techniques to isosurfaces provides even more insight into the characteristics of 3D vector fields. This has only recently become a feasible option. Figure 8 middle-right, shows a velocity isosurface of $5.0 \mathrm{~m} / \mathrm{s}$ in the combustion chamber of the data set in Figure 2 with texture-based flow visualization applied. We can see that the flow has some of the swirling orientation that we are looking for. The application of texture advection provides a clear indication of the upstream and downstream nature of the flow that is not visible with an isosurface alone. In particular, the texture-based approaches help to point where the flow does not follow the ideal swirl pattern that the combustion chamber should encapsulate, in this case in the top-middle between the two intake ports.

We also note that caution is advised when interpreting the result of texture-based flow visualization on isosurfaces because the direction of the flow does not necessarily align with the isosurface itself. Different approaches to incorporating this normal component of the flow with respect to the isosurface in the visualization include: (1) varying the texture convolution filter [1] or the surface color opacity according to the cross flow [10] or the possibility of not projecting the velocity vectors onto the surface [20]. For a more detailed discussion on this topic we refer to previous literature [10].

Figure 1 left shows a hybrid visualization using direct colormapping, streamlines, a velocity isosurface, and texture-based flow visualization. We note that in order to combine the ISA implementation with streamlines, we must ensure a proper rendering order to the objects. All other objects such as streamlines must be rendered before applying texture synthesis so that the image overlay from ISA does not cover these other objects [9]. One perceptual problem with the result in Figure 1 left is occlusion. Figure 1 middle-left illustrates the use of a clipping plane to reveal occluded flow struc- 

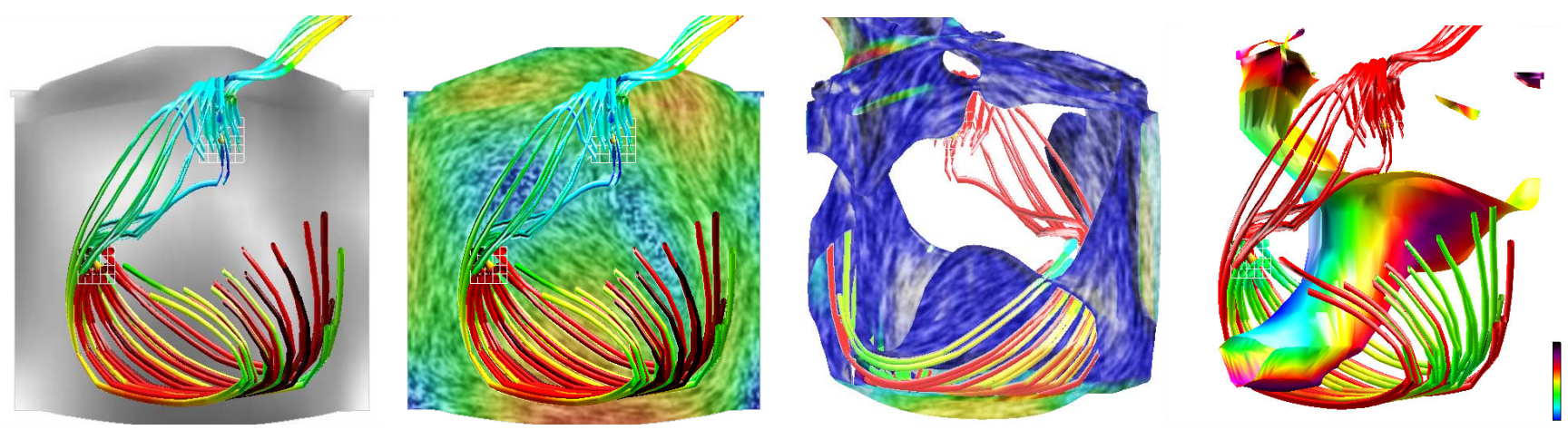

Figure 10: The visualization of 3D tumble flow: (left) geometric visualization using streamlines, (middle-left) geometric visualization streamlines accompanied by texture-based flow visualization on a 2D slice, (middle-right) geometric flow visualization using timelines, combined with texturebased flow visualization on a velocity isosurface, and (right) geometric flow visualization with streamlines and a pressure isosurface color-mapped with velocity magnitude.

tures such as the isosurface surrounding the intake ports.

Figure 10 middle-left shows a similar visualization with the addition of texture-based flow visualization applied to the slice. In this case, we can see that a subset of the 3D flow does reflect the characteristics of the tumble flow pattern. However, we can see that the axis of flow rotation is off-center. Instead of the axis pointing straight out along the $z$ axis, it is at an angle pointing down and to the left. It looks like a hybrid of the swirl and tumble flow patterns. Figure 10 middle-right shows a texture-based flow visualization applied to a velocity isosurface of $7.5 \mathrm{~m} / \mathrm{s}$ combined with $3 \mathrm{D}$ streamlines. In this case we have chosen a view from the back in order to reduce occlusion. The streamlines also verify that the flow exhibits a spatial component normal to the isosurface because they pass through the isosurface near the bottom.

The choice of isovalue is important when using isosurfaces to visualize the flow motion. The users rely on a priori knowledge of the data set from CFD experience in addition to some trial and error in order to obtain insightful results. The isosurface in Figure 10 middle-right is in fact not optimal because of its shape. The complexity of the isosurface does not lend the user an intuitive interpretation. That is why we chose another approach in Figure 10 right which shows an isosurface of uniform pressure-80,500 $\mathrm{Pa}$ (pascals). This result combines two geometric flow visualization methods namely isosurfaces and streamlines. This isosurface helps us to visualize the tumble flow's axis of rotation even more clearly than the streamlines alone. We may choose other CFD attributes from which to compute isosurfaces useful for visualization. Another popular attribute choice is that of spray equivalence ratio. The spray equivalence ratio is made of mass of air over mass of fuel. Thirteen parts of air for each part of fuel equals a spray equivalence ratio of unity. A ratio in the range of 0.7-1.4 represents an ignitable mixture. We use isovalues in this range to help track where the ignitable mixture is located. Figure 1 right combines three tools into a hybrid visualization: (a) texture-based flow visualization on a slice, (b) the pressure isosurface color-mapped according to velocity magnitude and, (c) 3D streamlines.

We have also looked at 3D texture-based flow visualization based on a 3D IBFV implementation [16] and a programmable graphics hardware implementation [23] in the context of investigating swirl and tumble flow. We generally find perceptual problems to be a great challenge with this approach. Similar to the resampling approach, a trade-off must be made between coverage of the flow domain and visibility of the flow. This is one reason why a hybrid of geometric and texture-based techniques is useful. Additional challenges for 3D texture-based approaches still remain implementation and efficiency issues because all the simulation results we present are on unstructured, adaptive resolution grids.

\section{Discussion}

We have investigated two typical flow patterns from CFD using three classes of flow visualization techniques commonly available in $2 \mathrm{D}, 2.5 \mathrm{D}$, and $3 \mathrm{D}^{1}$. Our side-by-side comparison of each flow visualization category illustrates that each has its respective advantages and disadvantages. Direct flow visualization techniques are intuitive, easy to implement, common, and insightful. The direct flow visualization techniques are useful in highlighting extremal CFD simulation data values on surfaces. However, direct approaches may not communicate flow evolution very clearly and are often more difficult to apply in 3D. And important features can be missed if the sampling rate for a glyph-based representation is not high enough.

Geometric methods are also intuitive, provide insight, and can be applied to 2D, 2.5D, and 3D vector data. They also sometimes indicate flow direction including the upstream and downstream direction of the flow. Geometric techniques are useful for gaining insight into the location of the axis of rotation for both the swirl and tumble flow patterns while texture-based techniques provided useful enhancements to these results (Figures 8 and 10). However, the drawback with these approaches is generally that of placement. Important features may be overlooked because they do not provide complete coverage of the flow domain.

Texture-based approaches share advantages with both direct and geometric techniques by providing complete coverage and showing the direction of the flow everywhere. However, they are difficult to apply in 3D. The geometric and texture-based techniques applied to surfaces were very good at pointing out where the ideal swirl flow pattern was not being met (Figure 6) in the CFD simulation model. The geometric techniques applied to slices and surfaces were also suitable for highlighting specific topological features of the flow (Figures 5 and 7) whereas the texture-based approaches were very helpful by insuring that these topological features were not initially overlooked. Figure 11 summarizes some of the trade-offs that are made when visualizing swirl and tumble motion. For example, The more dense the visualization, generally the more difficult is to perceive the result. Thus a trade-off is often made between these two factors. Also a trade-off may is made between spatial coherence and spatial dimensionality because results in 3D often contain many overlapping parts. As a result of these trade-offs, the flexible combinations of approaches offered by our system are good alternatives.

\footnotetext{
${ }^{1}$ For supplementary material including high resolution images and MPEG animations, please visit:

http://www.vrvis.at/ar3/pr2/swirl-tumble/
} 


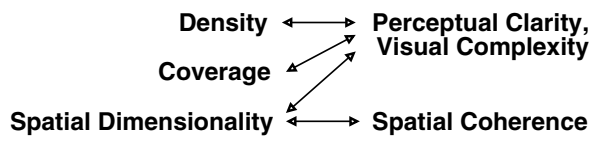

Figure 11: In general, trade-offs are made between the density, coverage, and spatial dimensionality of the visualization with that of perceptual clarity, visual complexity, and spatial coherence.

\section{CONClusions ANd Future Work}

In general, the optimal flow visualization technique depends on the needs of the user and the nature of vector field. For example, visualizing swirl flow using 3D streamlines is easier than for the case of tumble motion. We are able to emphasize and communicate different characteristics of the flow with different tools. Also, some methods are better for visualizing 2D flow rather than 3D flow. Hence, a range of tools is required to help us analyze CFD simulation data. Although we have focused on two specific flow patterns from CFD, we believe what we present here to be applicable to more general cases.

Future work includes the application of these three classes of flow visualization techniques to the investigation of motion associated with cooling jackets. The geometry and flow patterns associated with cooling jackets are generally even more complicated than that of swirl and tumble flow within a cylinder. We suspect that techniques which apply to the surface may be especially important since this type of geometry has a very high surface area. Also, since cooling jackets may contain many thin pieces in their geometry, visualization via slicing does not show as much spatial coherency as via surfaces. Three dimensional techniques may also prove to be very useful because cooling jackets are often characterized by components with thin volumes. Hence, texture-based flow visualization techniques applied in 3D may result in fewer perceptual issues.

\section{ACKNOWLEDGEMENTS}

The authors thank all those who have contributed to this research including AVL (www.avl.com), the Austrian government research program K plus (www.kplus.at through funding), and the Landesstiftung Baden-Württemberg in Germany. All CFD simulation data shown is courtesy of AVL. We also thank Alexandru Telea and Jarke J. van Wijk for providing us with their 3D IBFV application.

\section{REFERENCES}

[1] H. Battke, D. Stalling, and H.C. Hege. Fast Line Integral Convolution for Arbitrary Surfaces in 3D. In Visualization and Mathematics, pages 181-195. Springer-Verlag, 1997.

[2] S. Bryson and C. Levit. The Virtual Wind Tunnel. IEEE Computer Graphics and Applications, 12(4):25-34, July 1992.

[3] B. Cabral and L. C. Leedom. Imaging Vector Fields Using Line Integral Convolution. In Poceedings of ACM SIGGRAPH 1993, Annual Conference Series, pages 263-272. ACM Press / ACM SIGGRAPH, 1993.

[4] J. L. Helman and L. Hesselink. Representation and Display of Vector Field Topology in Fluid Flow Data Sets. IEEE Computer, 22(8):2736, August 1989.

[5] J. L. Helman and L. Hesselink. Visualizing Vector Field Topology in Fluid Flows. IEEE Computer Graphics and Applications, 11(3):3646, May 1991.

[6] B. Jobard, G. Erlebacher, and Y. Hussaini. Lagrangian-Eulerian Advection of Noise and Dye Textures for Unsteady Flow Visualization. IEEE Transactions on Visualization and Computer Graphics, 8(3):211-222, 2002.

[7] B. Jobard and W. Lefer. Creating Evenly-Spaced Streamlines of Arbitrary Density. In Proceedings of the Eurographics Workshop on
Visualization in Scientific Computing '97, volume 7. Eurographics, Springer-Verlag, 1997.

[8] R. S. Laramee, H. Hauser, H. Doleisch, F. H. Post, B. Vrolijk, and D. Weiskopf. The State of the Art in Flow Visualization: Dense and Texture-Based Techniques. Computer Graphics Forum, 23(2):203221, June 2004.

[9] R. S. Laramee, B. Jobard., and H. Hauser. Image Space Based Visualization of Unsteady Flow on Surfaces. In Proceedings IEEE Visualization '03, pages 131-138. IEEE Computer Society, 2003.

[10] R. S. Laramee, J. Schneider, and H. Hauser. Texture-Based Flow Visualization on Isosurfaces from Computational Fluid Dynamics. In Data Visualization, The Joint Eurographics-IEEE TVCG Symposium on Visualization (VisSym '04), pages 85-90,342. Eurographics Association, 2004

[11] F. H. Post, B. Vrolijk, H. Hauser, R. S. Laramee, and H. Doleisch. Feature Extraction and Visualization of Flow Fields. In Eurographics 2002 State-of-the-Art Reports, pages 69-100. The Eurographics Association, 2-6 September 2002.

[12] F. H. Post, B. Vrolijk, H. Hauser, R. S. Laramee, and H. Doleisch. The State of the Art in Flow Visualization: Feature Extraction and Tracking. Computer Graphics Forum, 22(4):775-792, Dec. 2003.

[13] A. Sanna, B. Montrucchio, and R. Arinaz. Visualizing Unsteady Flows by Adaptive Streaklines. In WSCG 2000 Conference Proceedings, pages 84-91, 2000.

[14] G. Scheuermann, H. Burbach, and H. Hagen. Visualizing Planar Vector Fields with Normal Component Using Line Integral Convolution. In Proceedings IEEE Visualization '99, pages 255-262. IEEE Computer Society, 1999.

[15] M. Schulz, F. Reck, W. Bartelheimer, and T. Ertl. Interactive Visualization of Fluid Dynamics Simulations in Locally Refined Cartesian Grids. In Proceedings IEEE Visualization '99, pages 413-416, 1999.

[16] A. Telea and J. J. van Wijk. 3D IBFV: Hardware-Accelerated 3D Flow Visualization. In Proceedings IEEE Visualization '03, pages 233-240. IEEE Computer Society, 2003.

[17] G. Turk and D. Banks. Image-Guided Streamline Placement. In SIGGRAPH 96 Conference Proceedings, Annual Conference Series, pages 453-460. ACM SIGGRAPH, Addison Wesley, August 1996.

[18] J. J. van Wijk. Spot noise-Texture Synthesis for Data Visualization. In Thomas W. Sederberg, editor, Computer Graphics (Proceedings of ACM SIGGRAPH 91), volume 25, pages 309-318. ACM, 1991.

[19] J. J. van Wijk. Image Based Flow Visualization. ACM Transactions on Graphics, 21(3):745-754, 2002.

[20] J. J. van Wijk. Image Based Flow Visualization for Curved Surfaces. In Proceedings IEEE Visualization '03, pages 123-130. IEEE Computer Society, 2003.

[21] V. Verma, D. Kao, and A. Pang. A Flow-guided Streamline Seeding Strategy. In Proceedings IEEE Visualization 2000, pages 163-170, 2000.

[22] D. Weiskopf, G. Erlebacher, and T. Ertl. A Texture-Based Framework for Spacetime-Coherent Visualization of Time-Dependent Vector Fields. In Proceedings IEEE Visualization '03, pages 107-114. IEEE Computer Society, 2003.

[23] D. Weiskopf and T. Ertl. GPU-Based 3D Texture Advection for the Visualization of Unsteady Flow Fields. In WSCG 2004 Conference Proceedings, Short Papers, pages 259-266, February 2004. 